\title{
UNA PROPOSTA DI RICERCA MULTIDISCIPLINARE PER L'EUROPA E IL BACINO DEL MEDITERRANEO
}

\author{
GIULIA VERTECCHI \\ gvertecchi@gmail.com \\ Aix-Marseille Univ, CNRS, MMSH, A*MIDEX, AIX-EN-ProvenCE \\ http://dx.doi.org/10.25267/Riparia_sup.2018.11.02
}

\begin{abstract}
Qualche anno fa il Consiglio Europeo in un documento pubblicato nel 2014 sull'Official Journal of the European Union, aveva invitato i paesi membri a investire in maniera più decisa nell'ambito delle proprie politiche nazionali sul patrimonio culturale, inteso come risorsa strategica per garantire una crescita sostenibile e inclusiva dell'Europa. Per chiarire le motivazioni della necessità di investimenti sulla valorizzazione del patrimonio culturale, il Consiglio Europeo ne precisava la definizione:
\end{abstract}

«cultural heritage consists of the resources inherited from the past in all forms and aspects - tangible, intangible and digital (born digital and digitized), including monuments, sites, landscapes, skills, practices, knowledge and expressions of human creativity, as well as collections conserved and managed by public and private bodies such as museums, libraries and archives. It originates from the interaction between people and places through time and it is constantly evolving. These resources are of great value to society from a cultural, environmental, social and economic point of view and thus their sustainable management constitutes a strategic choice for the $21^{\text {st }}$ century» ${ }^{1}$.

Il riconoscimento del patrimonio culturale come risorsa strategica e l'adozione di un'ampia definizione che richiama e sviluppa le indicazioni dell'UNESCO${ }^{2}$, segnano una linea di orientamento ben precisa nella politica dell'Unione Europea anche per quanto riguarda lo stanziamento dei fondi dedicati alla ricerca. Da questi presupposti nasce infatti il programma Horizon2020 (2014-2020) il più grande programma europeo dedicato al finanziamento dell'innovazione e della ricerca. Diversamente da quanto era accaduto per i precedenti programmi europei, come il $7^{\circ} \mathrm{WP}$, Horizon2020 non dedica una linea specifica di finanziamento alle scienze umane e ai beni culturali, ma pone le scienze umane e sociali (SSH) come tema trasversale a tutto il programma. Obiettivo di Horizon2020 è di integrare le scienze umane e sociali all'interno di diverse linee di finanziamento in modo tale da valorizzare il loro potenziale come chiave di lettura per risolvere le sfide complesse di una società in continua evoluzione. D'altra parte il patrimonio culturale non è semplice oggetto di analisi e ricerca delle singole discipline umanistiche, ma il suo studio e la sua promozione coinvolgono anche importanti politiche pubbliche come quelle legate allo sviluppo regionale, al turismo, all'ambiente, all'istruzione, alla coesione sociale, all'agricoltura. Di conseguenza le proposte da sottoporre al finanziamento di Horizon2020 devono inevitabilmente includere la dimensione sociale, economica, istituzionale, storica e culturale nell'affrontare i problemi posti dai cambiamenti della società e dell'ambiente. Horizon2020 infatti sostiene progetti basati sull'internazionalizzazione e la multidisciplinarità e in particolare sull'associazione tra discipline appartenenti alle scienze umane (storia, economia, arte, archeologia ecc.) e

${ }^{1}$ Official Journal of the European Union, C 183/36, 14.6.2014.

$2 \mathrm{http} / / /$ www.unesco.org/new/en/culture/themes/illicit-trafficking-of-culturalproperty/unesco-database-of-national-cultural-heritage-laws/frequently-askedquestions/definition-of-the-cultural-heritage/ 
discipline appartenenti alle cosiddette scienze dure (scienze naturali, ambientali, tecnologiche, fisiche ecc.), perché è attraverso l'interazione tra discipline diverse che emergono le soluzioni più innovative per una crescita intelligente, sostenibile e inclusiva dell'Europa ${ }^{3}$.

Il progetto CIRILI «City, River, Littoral. The Extension of Transportation Infrastructure in Delta Areas» si inserisce pienamente nel quadro sopra delineato sia perché abbraccia molteplici discipline di ambiti diversi (dall'archeologia e la storia, alle scienze naturali e ambientali), sia perché coinvolge accanto ad una rete accademica di ricercatori di prestigiose istituzioni internazionali, anche gli attori della politica locale, la società civile e le imprese. L'origine del progetto CIRILI risale a qualche anno fa, quando un gruppo di ricercatori, già da tempo impegnato nello studio delle problematiche sociali, urbanistiche, storiche e ambientali legate al delta del Tevere, ha ritenuto utile comparare i risultati delle proprie indagini con quelli di altri ricercatori che studiavano territori deltizi urbanizzati situati nel bacino del Mediterraneo. Lo studio del territorio posto alla foce del Tevere, tuttora in continua evoluzione e con una storia millenaria, ha messo in luce infatti alcune problematiche complesse, tra le quali quella legata all'impatto ambientale dell'espansione dell'aeroporto di Fiumicino in un'area caratterizzata da una forte urbanizzazione e dalla presenza di un patrimonio archeologico e paesaggistico di estremo valore (Ostia antica, la Necropoli di Porto all'Isola Sacra, il complesso monumentale dei porti imperiali di Claudio e Traiano). Il progetto CIRILI quindi nasce dall'esigenza di costituire una rete di ricercatori e dalla necessità di prendere in considerazione non solo lo studio storico sulla lunga durata, ma anche l'impatto del sistema di trasporti in queste aree litoranee, tra fiume, terra e mare. Proprio queste due esigenze hanno spinto nel 2014 a proporre il progetto CIRILI per il finanziamento nell'ambito di una COST Action" nella linea "Transport and Urban Development". Tuttavia l'asse "Transport and Urban Development", rappresentando solo uno degli aspetti di CIRILI, non permetteva di sviluppare pienamente il progetto molto più ambizioso: in particolare le tematiche legate al patrimonio culturale e alla dimensione storica del territorio non trovavano uno spazio adeguato in un asse dedicato soprattutto ai trasporti e dunque la COST Action si rivelava una scelta non del tutto soddisfacente ${ }^{5}$.

L'avvio del progetto CIRILI nel 2015 è stato possibile grazie al programma RAMSES 6 finanziato dalla fondazione A*MIDEX nell'ambito delle iniziative di eccellenza dell'Università di Aix-Marseille. Il progetto CIRILI, uno dei 5 Work Packages di RAMSES, è stato coordinato e diretto dall'École Française de Rome in piena autonomia e ciò ha permesso non solo di raggiungere i risultati previsti, ma ha anche posto le basi per uno sviluppo futuro in sintonia con gli obiettivi indicati dall'Europa e in particolare con quelli di Horizon2020.

\footnotetext{
3 «smart, sustainable and inclusive growth» è uno degli obiettivi strategici di Europa 2020.

4 http://www.cost.eu/COST_Actions : "COST Actions are a flexible, fast, effective and efficient networking instrument for researchers, engineers and scholars to cooperate and coordinate nationally funded research activities. COST Actions allow European researchers to jointly develop their own ideas in any science and technology field".

${ }^{5}$ CIRILI ha superato la prima fase della selezione COST, ma non la seconda proprio perché il progetto oltrepassava $i$ limiti tematici indicati dalla linea "Transport and Urban Development".

${ }^{6}$ http://labexmed.mmsh.univ-aix.fr/international/ramses/amidex/Pages/default.aspx
} 


\section{Obiettivi e risultati del progetto CIRILI}

Obiettivo del progetto CIRILI è uno studio comparativo di territori deltizi urbanizzati collocati attorno a quattro fiumi nel bacino del Mediterraneo: il Guadalquivir, il Nilo, il Rodano e il Tevere. Si tratta di territori estremamente complessi caratterizzati da una storia millenaria e stratificata che ha lasciato le sue tracce tangibili in un patrimonio culturale e ambientale di inestimabile valore. Si tratta però anche di territori particolarmente esposti a sfide difficili da affrontare, come i cambiamenti climatici (innalzamento del livello del mare), diverse forme di inquinamento, l'urbanizzazione, la pressione turistica. Le tappe del progetto sono state scandite da tre incontri internazionali e da un atelier dottorale che hanno visto la partecipazione, accanto ai ricercatori e agli studenti, di attori coinvolti nella gestione e amministrazione di questi territori. I principali temi affrontati in questi incontri riguardano: il ruolo delle vie d'acqua per la circolazione delle merci, l'impatto sul territorio delle strutture legate al trasporto (aeroporti, porti, ferrovie, strade), e lo studio delle modalità per rendere accessibile ai cittadini il patrimonio naturalistico e archeologico di queste aree.

La navigabilità di queste quattro importanti vie d'acqua è uno degli aspetti più interessanti affrontati dallo studio in una prospettiva comparativa e di lungo periodo: il Guadalquivir, il Nilo, il Rodano e il Tevere sono fiumi navigabili sin dall'antichità, ma il Nilo e il Tevere non hanno conservato il ruolo di asse commerciale che avevano nell'antichità, a causa delle trasformazioni morfologiche subite dal territorio e, almeno per quanto riguarda il Tevere, anche a causa delle caratteristiche stesse del fiume. Attualmente gli aspetti normativi che regolano il bacino del fiume Tevere e il distretto dell'Appennino centrale sono numerosi e complessi e si scontrano con un corso d'acqua caratterizzato dall'irregolarità della sua portata, che attraversa diversi territori con forti dislivelli [G. Cesari] $]^{7}$. Inoltre l'evoluzione della linea di riva in prossimità delle foci del Tevere è ben documentata da diverse cartografie storiche e permette di stabilire che, nel complesso, tra il Rinascimento e l'inizio del XX secolo si è prodotta una progradazione dell'apice deltizio di circa $3 \mathrm{~km}$. Questa tendenza evolutiva sembra essersi invertita dalla metà del XX secolo in parte per l'innalzamento del livello del mare e in parte per il continuo decremento del trasporto solido in sospensione delle acque, successivo alla costruzione delle dighe lungo il corso del Tevere [P. Bellotti] ${ }^{8}$.

Diversamente dal Tevere, il Guadalquivir e il Rodano con i porti di Siviglia e di Arles hanno mantenuto una forte funzione economica e fanno oggi parte della grande rete di trasporto fluvio-marittima europea. Il Guadalquivir era un fiume più facilmente navigabile in epoca romana rispetto alle epoche successive, sebbene periti e ingegneri romani, come testimoniato dai resti archeologici, si erano già dovuti confrontare con alcuni problemi legati all'irregolarità della corrente [L. del Moral Ituarte] ${ }^{9}$. Nel corso dei secoli l'estuario del Guadalquivir ha subito dei cambiamenti morfologici importanti che hanno reso in alcuni periodi molto difficile la navigazione. In particolare in età moderna la navigabilità del fiume rappresentava spesso una sfida per le imbarcazioni che, provenendo dal mare, dovevano risalire il suo corso: il più grande ostacolo da superare infatti era costituito dalla barra dell'estuario, la celebre

\footnotetext{
7 Vedi testo in questo stesso volume e anche http://cirili.hypotheses.org/84

8 Vedi testo in questo stesso volume e anche http://cirili.hypotheses.org/45

${ }_{9}^{9}$ Vedi testo in questo stesso volume e anche http://cirili.hypotheses.org/489
} 
barra di Sanlúcar. La morfologia di questo tratto finale del fiume era profondamente cambiata rispetto all'epoca romana, al punto che il tasso di naufragio era altissimo. E' possibile seguire l'evoluzione nel tempo di questi cambiamenti grazie ad un attento studio e confronto tra le carte nautiche attuali con la documentazione storica costituita soprattutto da piante e mappe $[\text { L. Ménanteau }]^{10}$. Oggi le navi passano in un canale totalmente artificiale che si è sovrapposto alla barra di Sanlúcar e che ha permesso di superare i problemi legati alle dimensioni e alla profondità della barra.

Il territorio del basso Guadalquivir è molto vasto e diviso geograficamente in tre aree: la zona costiera a sud di Cadice, quella più a nord di Sanlúcar di Barrameda e quella più interna di Siviglia, mentre, risalendo ancora oltre il corso del fiume, la navigazione diventa più complessa e da fluvio-marittima diventa fluviale con la conseguente necessità di utilizzare apposite imbarcazioni [J. Ramón Barros Caneda] ${ }^{11}$. Attualmente il porto fluvio-marittimo di Siviglia è una realtà economica importante per lo sviluppo del territorio che necessita di infrastrutture moderne per garantire il suo funzionamento. Oltre alle infrastrutture portuali, anche l'uso dell'acqua del fiume per l'irrigazione, la creazione di campi da golf e l'urbanizzazione sono fattori che influiscono notevolmente sull'equilibrio ambientale dell'ampia regione del basso Guadalquivir dove, tra l'altro, si trova anche il Parco Nazionale di Doñana, caratterizzato da una biodiversità unica in Europa [A. Villalpando $]^{12}$. La gestione dell'uso dell'acqua del Guadalquivir crea dunque situazioni conflittuali molto forti che vedono contrapporsi l'Autorità Portuale di Siviglia - che vorrebbe aumentare la profondità del fiume - la Rete Natura 2000 di cui fa parte il Parco di Doñana, la Confederación Hidrográfica del Guadalquivir, diverse commissioni scientifiche istituite per produrre studi di fattibilità sul dragaggio del fiume e l'Europa che non vuole concedere finanziamenti senza la garanzia di uno sviluppo sostenibile [L. del Moral Ituarte].

Ben diversa la situazione del Rodano dove il porto fluvio-marittimo di Arles è perfettamente integrato nel contesto ambientale. Il porto, di piccole dimensioni, può accogliere imbarcazioni provenienti dal mare fino alla portata di 3000 tonnellate e registra un flusso di merci annuale di 500.000 tonnellate, circa un decimo del movimento delle merci del porto di Siviglia ${ }^{13}$. Lo sviluppo delle infrastrutture del porto (costituite attualmente da 6 ha di superfici di stoccaggio, aree di manutenzione, da una banchina sud di $220 \mathrm{~m}$ e una nord di $180 \mathrm{~m}$ ), rientra in un piano nazionale elaborato di concerto con le realtà locali, tenendo conto delle caratteristiche del territorio e della necessità di preservare il patrimonio ambientale e culturale. Di conseguenza la pianificazione della costruzione del porto, secondo un piano condiviso dalla politica nazionale e locale, non ha generato quelle situazioni conflittuali che si riscontrano per il Guadalquivir, il Nilo e il Tevere. Bisogna inoltre sottolineare che il trasporto fluviale è un ottimo esempio di sviluppo sostenibile perché permette di trasportare merci pericolose e ingombranti attraversando il paesaggio con un impatto ambientale di gran lunga inferiore al trasporto su gomma o

\footnotetext{
${ }^{10}$ Vedi testo in questo stesso volume e anche http:// cirili.hypotheses.org/60

11 Vedi testo in questo stesso volume e anche http://cirili.hypotheses.org/347; per la navigazione fluviale sul Guadalquivir vedi http://cirili.hypotheses.org/colloquerome e la registrazione audio dell'intervento di I. Bestué Cardiel, Los proyectos de navegabilidad del Guadalquivir

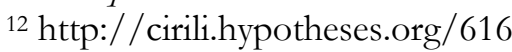

${ }^{13} \mathrm{http}$ ///www.puertos.es/es-es/estadisticas/Paginas/estadistica_mensual.aspx
} 
su ferro [B. Ponchon $]^{14}$. Anche il territorio del delta del Rodano, come quello del Guadalquivir, è caratterizzato dalla presenza di un'area naturale protetta che costituisce il Parco Naturale Regionale della Camargue: l'organismo parco, qui, non svolge soltanto un'azione di gestione delle risorse ambientali (uso dell'acqua per l'agricoltura, censimento della fauna e della flora), ma promuove il turismo e dialoga con le altre istituzioni locali con la finalità di coinvolgere la società civile e sostenere iniziative in favore dell'educazione all'ambiente [E. Rouquette-C. Ser] ${ }^{15}$. Inoltre il moderno porto fluvio-marittimo di Arles sorge solo poco più a nord dell'antica città romana che, allora come oggi, deve gran parte del suo sviluppo economico proprio al Rodano. Da circa 25 anni il Rodano è il territorio di scavo più produttivo della città $\mathrm{e}$ le migliaia di oggetti che ha permesso di portare alla luce in un ottimo stato di conservazione, grazie alle acque dolci del fiume, costituiscono dei tasselli importanti per ricostruire la vita economica e le attività di un porto con una lunga continuità d'uso nei secoli. Nel 2009 il Consiglio Generale del dipartimento Bouches-du-Rhône (Conseil Général des Bouches-du-Rhône) ha finanziato una prima mostra dedicata ai ritrovamenti nel Rodano. Questa mostra ha entusiasmato il pubblico al di là di ogni previsione, mostrando come l'archeologia abbia forti ripercussioni sulla società e sull'economia e come essa non sia soltanto una disciplina specialistica che riguarda l'ambito accademico. E' ancora grazie al sostegno delle istituzioni e ad un grande lavoro di équipe che è stato possibile riportare alla superficie un'imbarcazione denominata Arles-Rhône3. Si tratta di un rinvenimento del tutto eccezionale di un'imbarcazione fluviale gallo-romana lunga $31 \mathrm{~m}$, praticamente intera e completa del suo carico: tutte le operazioni di recupero del relitto, di restauro, di allestimento di una nuova ala del Musée Départemental Arles Antique, costruita e progettata ad hoc per poter esporre il relitto, sono state completate in pochissimo tempo, meno di tre anni [C. Sintès, A. Charron, S. Marlier, D. Djaoui $]^{16}$.

Molto diverso, almeno fino ad oggi, il destino del Museo delle Navi romane di Fiumicino, che si trova sull'asse stradale che collega le strutture aeroportuali alla rotatoria, punto di smistamento del traffico automobilistico tra l'aeroporto e gli insediamenti di Fiumicino, Isola Sacra e Ostia. Il padiglione iniziale del museo venne costruito e usato come deposito e laboratorio di restauro per i relitti delle navi, rinvenuti tra il 1958 e il 1965 durante i lavori di costruzione dell'aeroporto internazionale Leonardo da Vinci: il vero e proprio museo, costruito a partire dal 1966, fu aperto soltanto tredici anni dopo, nel 1979. Chiuso nel 2002 per motivi strutturali è tutt'oggi non accessibile al pubblico, anche se la sua riapertura sembra ormai vicina, grazie anche ad un progetto che prevede il recupero della struttura e il suo successivo ampliamento per aumentare la capacità espositiva e comunicativa del museo [S. Borghini, C. Collettini, M.T. Donzelli, R. Sebastiani] $]^{17}$. Il Museo delle Navi dunque sorge vicino al principale hub aeroportuale italiano, l'aeroporto Leonardo da Vinci (con un traffico di oltre 40 milioni di passeggeri l'anno), che in questo caso coincide con quello che fu il più grande hub portuale dell'antichità situato nell'area del delta del Tevere: il sistema portuale romano, con i porti di Claudio e Traiano

14 http:// cirili.hypotheses.org/331
15 http:// cirili.hypotheses.org/356
16 Vedi testo in questo stesso volume e http://cirili.hypotheses.org/283 ;
http://cirili.hypotheses.org/253 ; sul servizio pedagogico del museo
http:// cirili.hypotheses.org/632
17 http:// cirili.hypotheses.org/315; S. Borghini, C. Collettini, M.T. Donzelli, R. Sebastiani,
Dal museo al museo: il progetto del nuovo Museo delle Navi, «Forma Urbis», dicembre 2016, p. 23-26. 
(Portus), fu il principale porto marittimo della Roma imperiale e svolse un ruolo fondamentale nell'approvvigionare la Capitale di alimenti e merci provenienti da tutto il bacino del Mediterraneo tra la metà del I e il VI secolo d.C.

L'area archeologica di Portus - in seguito alla recente riforma confluita nel Parco Archeologico di Ostia Antica - è oggi un centro internazionale di ricerca storicoarcheologica e di formazione al quale lavorano, da anni e a diverso titolo, gruppi di studio francesi (École Française de Rome, dalla metà degli anni 2000), inglesi (British School at Rome, dal 1998) e italiani (Ministero dei Beni Culturali con le sue soprintendenze locali): la fruizione al pubblico del parco archeologico è ancora fortemente limitata anche se negli ultimi anni la situazione è notevolmente migliorata. I risultati e le ricadute della ricerca hanno alimentato i progetti didattici, impostati e realizzati con un accordo tra il Ministero dei Beni Culturali, la Fondazione Benetton, Aeroporti di Roma, il Comune di Fiumicino, la rete scolastica Eco-Schools [R. Sebastiani] ${ }^{18}$. Il Parco archeologico naturalistico del Porto di Traiano si estende su una superficie di 33 ettari e rappresenta un importante sistema da salvaguardare per lo sviluppo di ecosistemi e per la biodiversità. Anche per il delta del Tevere, dunque, come per il Guadalquivir e il Rodano, l'area deltizia è caratterizzata dalla presenza di una riserva naturalistica di particolare pregio protetta dalle normative nazionali ed europee. Nel caso del Parco del Porto di Traiano, a differenza del Parco Nazionale di Doñana e di quello della Camargue, il sito naturalistico coincide con quello archeologico costituendo un complesso sistema di grande importanza storica, architettonica e paesaggistica [G. Strano $]^{19}$. Le preoccupazioni sul futuro utilizzo di questo prezioso spazio pubblico generano una forte conflittualità tra tutte le numerose amministrazioni, società private e movimenti associativi di cittadini interessati alla valorizzazione e all'utilizzo dell'area [D. Rivière ${ }^{20}$. D'altra parte tutto il territorio compreso nella piana del Tevere tra Roma e il mare è caratterizzato da un sistema ambientale fragile che ha subito pesanti trasformazioni e opere di urbanizzazione e che i regimi di pianificazione articolati non riescono a gestire per un intreccio e conflitto di competenze [K. Lelo, A.L. Palazzo] ${ }^{21}$.

Anche l'area del delta del Nilo presenta un sistema ambientale fragile che ha subito e continua a subire pesanti trasformazioni, ma con un impatto più distruttivo sul patrimonio e sul paesaggio rispetto alla situazione del Guadalquivir, del Rodano e del Tevere. A ciò si aggiunge che la rivoluzione del 25/1/2011 ha causato non solo una drastica riduzione del turismo e delle conseguenti entrate economiche, ma anche ricorrenti manifestazioni di ostilità nei confronti del patrimonio (spoliazione del museo del Cairo, dei depositi e dei siti archeologici): questi avvenimenti hanno indotto l'ICOM (International Council of Museums) a pubblicare una "Liste Rouge" dei beni culturali egiziani in pericolo. In questa ottica di tutela del patrimonio, i musei rivestono infatti un ruolo importante e per questo sono stati lo strumento principale di azione concreta nella politica del Supreme Council of Antiquities che nel 2005 ha programmato la costruzione o il rinnovamento di ben 33 musei [M.-C. Bruwier] ${ }^{22}$.

\footnotetext{
${ }^{18}$ R. Sebastiani, Portus : un paesaggio per il futuro, «Forma Urbis», dicembre 2016, p. 6-9.

${ }^{19}$ Vedi testo in questo stesso volume e http:/ / cirili.hypotheses.org/371

${ }^{20}$ Vedi testo in questo stesso volume e http:/ / cirili.hypotheses.org/504

${ }^{21}$ Vedi testo in questo stesso volume e http:// cirili.hypotheses.org/361

22 Vedi testo in questo stesso volume e http://cirili.hypotheses.org/643 ; http:// cirili.hypotheses.org/637
} 
Il patrimonio ambientale in generale, $\mathrm{ma}$ in particolare $\mathrm{i}$ resti archeologici sono minacciati dall'espansione urbana e dall'innalzamento del livello delle acque nell'area del delta del Nilo, al punto che, ad esempio, l'antica Thonis (Herakleion) si trova oggi a diversi chilometri di distanza dalla costa. Gli scavi condotti in campagne di archeologia subacquea sono dunque fondamentali per ricostruire e comprendere la storia del delta e della civiltà egiziana. Le indagini sottomarine nella zona della fortezza di Qaitbay ad Alessandria hanno permesso nel corso del tempo e grazie all'evoluzione delle tecniche di archeologia subacquea, di portare alla luce migliaia di frammenti che costituivano il famoso Faro di Alessandria, così come alcune statue che dovevano essere originariamente poste davanti alla Biblioteca alessandrina. Tuttavia i costi necessari per le complesse campagne di scavo e la grande quantità di reperti ancora sommersi rendono difficile far conoscere ai non addetti ai lavori i risultati del lavoro degli archeologi. E' soprattutto attraverso l'utilizzo delle tecniche di ricostruzione digitali e lo sviluppo di strumenti accessibili attraverso il web che sarà possibile valorizzare e rendere fruibile al grande pubblico questo importante patrimonio archeologico. [I. Hairy ${ }^{23}$.

A sud di Alessandria si estende la Mareotide, la campagna che si sviluppa attorno al lago Mariout: quest'area è un'interfaccia idrologica caratterizzata dal passaggio tra una zona desertica e una zona deltaica. Il Centre d'Études Alexandrines (CEAlex) lavora sulla Mareotide da più di 30 anni e dispone ormai di una gran mole di documentazione. Grazie ad un finanziamento dell'ANR (Agence Nationale de la Recherche) per il periodo 2013-2016, il CEAlex insieme ad altri partner egiziani e europei ha intrapreso il programma GEOMAR che ha l'obiettivo di realizzare una carta archeologica paleo-ambientale della Mareotide. Si tratta di uno studio inter- e pluridisciplinare che associa paleo ambientalisti, archeologi, topografi-cartografi e storici. La carta prodotta dal sistema GIS è anche uno strumento per la gestione dell'area che permette un'analisi spaziale e temporale dei dati cartografici, archeologici, bibliografici e dei sondaggi sul terreno [C. Shaalan, I. Awad, D. Dixneuf, Cl. Flaux, D. Kaniewski, N. Marriner, M.-D. Nenna, V. Pichot] ${ }^{24}$.

A causa dei lavori di bonifica condotti al principio del XX secolo e della crescente espansione urbana e industriale di Alessandria, il lago Mariout attualmente ha una dimensione di $\mathrm{km}$ 70x11, che rappresenta appena il 18\% della sua estensione nel 1801: il lago è utilizzato non solo come bacino di deflusso delle acque industriali, ma le acque del lago sono anche utilizzate per l'allevamento ittico, per l'irrigazione dei terreni agricoli, e per la coltura del sale. Il sistema GIS elaborato nell'ambito del progetto GEOMAR ha permesso ai ricercatori di localizzare e individuare 105 siti archeologici dei quali solo 13 classificati dal Ministry of State for Antiquities. L'integrità di questi siti è minacciata da diversi fattori, i più importanti dei quali sono: la non corretta determinazione dei limiti archeologici da parte del Ministry of State for Antiquities; i cambiamenti del livello dell'acqua del lago che hanno già causato l'inondazione di alcuni siti archeologici. Il progetto di pianificazione del governatorato di Alessandria (2005-2017) di dichiarare il lago Mariout come zona protetta, di prevedere la depurazione dell'acqua non sono stati ancora realizzati. D'altra parte la mancanza di cooperazione tra le diverse amministrazioni egiziane (governatorato, ministero dell'agricoltura, ambiente, antichità) impedisce di fatto di

${ }^{23} \mathrm{http}: / /$ cirili.hypotheses.org/693

24 Vedi testo in questo stesso volume e http://cirili.hypotheses.org/366 e http:// cirili.hypotheses.org/271 
mettere in atto una politica generale più efficace per la conservazione e la tutela del lago Mariout [I. Awad $]^{25}$.

Un aspetto al quale il CEAlex dedica particolare cura è il servizio pedagogico creato nel 2002 nell'ambito di un programma di cooperazione nel Mediterraneo. Le sue attività permettono agli abitanti di Alessandria di interessarsi al proprio patrimonio culturale, di introdursi ai mestieri dell'archeologo, di seguire i lavori scientifici e gli avanzamenti della ricerca, di visitare siti e monumenti della loro città e di aprirsi alle civiltà mediterranee. Infatti le attività per gli studenti delle scuole e gli avvenimenti culturali destinati al grande pubblico sono organizzati in partenariato con numerose istituzioni egiziane, francesi e straniere (non meno di 35 partner ogni anno). Molte di queste iniziative, collegate al Conseil Régional Provence-Alpes-Côtes d'Azur (PACA) sono organizzate nelle città di Marsiglia e Arles, permettendo di legare Alessandria ad un quadro mediterraneo e di presentare il patrimonio comune che possono condividere le diverse città [Ch. Gleize, M. Gawad] ${ }^{26}$.

$\mathrm{Da}$ quanto delineato in queste poche pagine, è evidente che l'interesse del progetto CIRILI nasce dalla comparazione e dalla dimensione internazionale. Infatti anche se il territorio deltizio di ciascuno di questi grandi fiumi presi in esame ha una propria storia e segue una sua specifica evoluzione nel tempo, è proprio dal confronto tra le quattro aree deltizie che si riesce a comprendere più a fondo il singolo caso studio e che nascono prospettive di ricerca di largo respiro per poter rispondere alle sollecitazioni delle politiche europee in tema di innovazione.

\section{Diffusione dei risultati della ricerca e prospettive future}

La diffusione dei risultati di una ricerca è una delle operazioni più delicate e a volte complesse che ogni ricercatore deve affrontare alla conclusione del proprio progetto. Nel caso di un progetto multidisciplinare come CIRILI, che si propone di costituire una rete di ricerca in grado di coinvolgere la società civile e di promuovere anche la formazione in materia di patrimonio culturale, l'attività di diffusione è per certi versi ancora più importante perché è parte integrante del successo del progetto. Proprio per questo motivo parallelamente all'organizzazione di tavole rotonde, convegni e scuole dottorali si è scelto sin dall'inizio di creare un blog [http://cirili.hypotheses.org] destinato in primo luogo ad accogliere tutti i materiali testuali e multimediali presentati negli incontri CIRILI.

La creazione del blog ha due obiettivi: il primo è quello di adottare le indicazioni dell'Europa in tema di Open Access che è una delle chiavi della cosiddetta «Responsible Research and Innovation» (RRI) per rispondere ai bisogni dei cittadini e coinvolgere il maggior numero possibile di attori sociali. Come si legge infatti nell'introduzione al Work Programme di Horizon2020, un punto essenziale sul quale l'Europa insiste è costituito dalla "communication, open access to research results and a new emphasis on data management". In questo senso il blog è uno strumento di comunicazione che ben si concilia con l'Open Access in quanto, sfruttando le potenzialità del web, fornisce il libero accesso ai dati della ricerca. Il secondo obiettivo del blog è quello di sperimentare una modalità di scrittura e di pubblicazione diversa rispetto a quelle tradizionalmente in uso nel mondo accademico: la versatilità del blog e la sua semplicità d'uso permettono di raggiungere

25 Vedi testo in questo stesso volume e http:/ / cirili.hypotheses.org/352

26 Vedi testo in questo stesso volume e http:/ / cirili.hypotheses.org/624 
un pubblico vario ed eterogeneo ridefinendo i rapporti tra scienza e società ${ }^{27}$. E' evidente che queste potenzialità del blog risultano preziose per il progetto CIRILI che ha tra $\mathrm{i}$ suoi obiettivi quello di coinvolgere un pubblico ampio, non solo accademico, in un dibattito su temi come la valorizzazione e la tutela del patrimonio archeologico e ambientale, l'urbanizzazione, la rete delle infrastrutture e dei trasporti.

Per conferire al blog «CIRILI. City, River, Littoral» una connotazione scientifica anche dal punto di vista formale ci si è avvalsi dell'opportunità di utilizzare la piattaforma «hypotheses.org» che, dalla sua creazione nel 2009, accoglie ormai diverse migliaia di blogs dedicati alle scienze umane e sociali. Hypothèses fa parte di un portale più vasto, OpenEdition, una vera e propria infrastruttura completa per l'edizione elettronica al servizio della diffusione delle pubblicazioni nelle scienze umane e sociali. OpenEdition, che è un'iniziativa pubblica senza fini di lucro proposta dal Centre pour l'édition électronique ouverte (Cléo), è sostenuta da diverse grandi istituzioni di ricerca e ha come principale missione la promozione dell'edizione elettronica ad accesso libero ${ }^{28}$. La piattaforma Hypothèses mette a disposizione non solo il supporto tecnico e la formazione gratuita per i suoi utenti, ma offre due ulteriori importanti vantaggi ai blogs che rispondono ai requisiti necessari per essere iscritti al catalogo OpenEdition ${ }^{29}$ :

1) grazie ad un accordo stipulato con la Bibliothèque Nationale de France (BnF), l'attribuzione di un numero di ISSN (International Standard Serial Number), ovvero l'identificativo unico caratteristico delle pubblicazioni periodiche che garantisce al blog anche la sua reperibilità nel catalogo delle pubblicazioni delle biblioteche;

2) l'indicizzazione di ogni singolo post nella banca dati ISIDORE, specializzata nelle scienze umane e sociali, che integra tipologie di fonti diverse (testi, metadati dei documenti, documenti multimediali) e fornisce ai blogs e al loro contenuto una maggiore visibilità nel mondo scientifico, ma anche più in generale una collocazione migliore nei motori di ricerca come Google.

Dato che il blog «CIRILI. City, River, Littoral» è nato assieme al progetto CIRILI e che il suo contenuto dipende dallo stato di avanzamento del progetto stesso, tutto il processo di indicizzazione ha richiesto qualche mese di tempo per il suo avvio. Tuttavia già da settembre 2015 CIRILI è iscritto al catalogo OpenEdition; a giugno 2016 ha ricevuto dalla Bibliothèque Nationale de France un permalink ${ }^{30}$ e il numero ISSN (2494-551X); dalla stessa data è nel catalogo online WorldCat ${ }^{31}$; da luglio 2016 è presente nella Directory of Open Access Scholarly Resources (ROAD) ${ }^{32}$.

\footnotetext{
27 M. Dacos, P. Mounier, Les carnets de recherche en ligne, espace d'une conversation scientifique décentrée, in «Lieux de savoir, T.2, Gestes et supports du travail savant», Albin Michel, pp. N/A, 2010, <HAL Id :sic_00439849>

${ }^{28}$ Di OpenEdition fanno parte anche una piattaforma dedicata alle riviste (Revues.org), una dedicata ai libri (OpenEdition Books) e un calendario online (Calenda) che annuncia migliaia di eventi accademici, cfr. https://fr.hypotheses.org/a-propos-dhypotheses

${ }^{29}$ Per i requisiti, cfr. http://maisondescarnets.hypotheses.org/2881

${ }^{30} \mathrm{http}: / /$ catalogue.bnf.fr/ark:/12148/cb45060067p

31 https://www.worldcat.org/title/city-river-littoral-cirili-lextension-des-infrastructures-detransport-en-zones-deltaiques/oclc/953088583\&referer=brief_results

32 http://road.issn.org/issn/2494-551X-city-river-littoral\#.WL1JlxCe9nt
} 
Come è noto, la caratteristica principale dei blogs è quella di essere organizzati sul principio della sola successione temporale, in modo tale che l'ultimo post pubblicato appaia sempre in primo piano richiamando il modello di scrittura tipico di un diario o di un giornale. Per guidare il lettore attraverso il contenuto del blog, «CIRILI. City, River, Littoral» è strutturato sulla base di una gerarchia di categorie e di etichette (tags), una parte delle quali è visibile dai due menu principali, mentre l'altra parte è accessibile tramite il menu a tendina «Catégories» nella barra di navigazione destra del blog. Categorie e etichette richiamano i temi (per es. Tevere, Rodano, Nilo, Guadalquivir) e le attività del progetto CIRILI (ColloqueRome, Table Ronde Arles, Coloquio Sanlúcar de Barrameda, Atelier doctoral Alexandrie, News). Questa classificazione dei posts attraverso categorie ed etichette permette di accedere alla lettura dei posts in base all'interesse e alla scelta del lettore e indipendentemente dall'ordine di pubblicazione che, come si è detto, segue il criterio cronologico. Il blog CIRILI è inoltre dotato di un campo per la ricerca libera e di un widget ISIDORE, ovvero una miniapplicazione denominata "Suggestions ISIDORE", che suggerisce ulteriori letture e approfondimenti in base all'argomento del post ${ }^{33}$, attingendo le informazioni direttamente dalla banca dati in scienze umane e sociali ISIDORE ${ }^{34}$.

Dall'analisi delle statistiche di consultazione del blog per il periodo gennaio-dicembre $2016^{35}$ emerge chiaramente che il bacino di lettori è molto più ampio rispetto a quello dei diretti partecipanti al progetto sia sotto il profilo quantitativo che geografico. Come è stato recentemente rilevato, il sistema di misurazione degli accessi ai blog sulla piattaforma "Hypothèses" sono in parte falsati dal fatto che non è possibile distinguere l'accesso umano da quello automatico di alcuni programmi molto diffusi nella rete (bots) ${ }^{36}$. Tuttavia il tasso di fedeltà dei visitatori, cioè il rapporto tra il numero delle visite e il numero dei visitatori, mostra che il contenuto del blog ha destato un interesse tale nel visitatore da indurlo a reiterare la visita. E' chiaro che nella pura logica della quantità che si è imposta sul web - ma che è bene ricordarlo non è sinonimo di qualità - la creazione di un profilo CIRILI su uno dei social media (per es. Twitter o Facebook) avrebbe con molta probabilità comportato un deciso aumento dei visitatori. Le ragioni per cui si è deciso di non aprire un profilo social sono essenzialmente due:

1) ogni partner del progetto CIRILI disponeva di propri canali di comunicazione già ampiamente consolidati e ha potuto diffondere le iniziative promosse da CIRILI all'interno della propria rete. Inoltre la costruzione di un adeguato profilo social avrebbe dato i suoi risultati e benefici per il progetto in un tempo più lungo rispetto a quello ben delimitato e piuttosto breve imposto dal finanziamento;

2) attraverso lo strumento del blog il progetto CIRILI si proponeva di sperimentare un sistema di scrittura, aperto anche al mondo non strettamente accademico, che permettesse la condivisione dei dati della ricerca. L'interfaccia del blog, piuttosto

\footnotetext{
${ }^{33}$ E' chiaro che perché i suggerimenti ISIDORE siano il più possibile pertinenti è necessario segnalare con un apposito codice alcune parole o frasi al momento della redazione del post. ${ }^{34} \mathrm{Il}$ vantaggio dell'indicizzazione in ISIDORE consiste anche nel fatto che questa banca dati è costruita sul linguaggio RDF e che quindi il suo contenuto può facilmente essere connesso ad altre risorse come NAKALA (un sistema di archiviazione dei dati della ricerca umanistica).

${ }^{35}$ https: $/ /$ logs.openedition.org/awstats.pl?month=all\&year $=2016 \&$ output $=$ main\&config $=\mathrm{cir}$ ilihypothesesorg\&lang $=$ fr\&framename $=$ index

${ }^{36} \mathrm{I}$. Mayeur, La communication scientifique directe vers un public élargi, «Revue française des sciences de l'information et de la communication», 11, 2017, p. 15.
} 
semplice nelle sue funzionalità di base, è teoricamente alla portata di tutti e consente a chiunque lo desideri di contribuire al blog, pubblicando autonomamente il proprio post, una volta che l'amministratore abbia creato il profilo utente necessario per l'autenticazione.

Nell'ambito del progetto CIRILI dunque il blog ha avuto la funzione di far confrontare una rete di ricercatori, composta per lo più di accademici, con la sfida della comunicazione al di fuori del proprio ambiente scientifico e in direzione dell'inclusione e della società partecipativa, come indicato dalle politiche europee in tema di ricerca e innovazione. Proprio l'esperienza del blog ha aperto nuove prospettive di sviluppo, perché ha permesso di rilevare la difficoltà di tradurre in pratica le indicazioni dell'Unione Europea quando la ricerca coinvolge progetti multidisciplinari, come è il caso di CIRILI. Infatti se è vero che l'Europa - in nome dell'open access e dell'inclusione - spinge verso la digitalizzazione di un'enorme quantità di documenti e dati (i cosiddetti big data) ${ }^{37}$, che sostiene grandi progetti come per es. «Europeana.eu» ${ }^{38}$, e che promuove la costituzione di consorzi come DARIAH-EU (Digital Research Infrastructure for the Arts and Humanities) ${ }^{39}$, è anche vero che per accedere a queste preziose risorse è indispensabile una discreta competenza digitale che non è comunemente diffusa a livello della società civile. Paradossalmente la digitalizzazione, se non accompagnata da un'adeguata formazione, non è affatto inclusiva e accessibile a tutti: esiste infatti un divario crescente tra una comunità accademica con competenze informatiche sempre più sofisticate e specialistiche, spesso tuttavia limitate al proprio ambito disciplinare, e la società civile che accede alle risorse sul web tramite i canali di comunicazione di massa (Facebook, Twitter e blog), ma che non possiede per lo più le competenze e gli strumenti critici per trarre beneficio dai grandi progetti di digitalizzazione europei.

A ciò si aggiunge che le numerose discussioni (giornalistiche ma anche accademiche) considerano Facebook, la rete, il web come se fossero una sola cosa dal punto di vista di ciò che le persone vi possono fare, mentre queste tecnologie permettono ognuna una varietà di usi e di pratiche sociali e comunicative molto diverse l'una dall'altra. Non ha senso infatti parlare di Facebook o di Twitter in generale perché i milioni di persone che li usano in tutto il mondo vi fanno le cose più disparate che sono del tutto inconfrontabili tra loro: l'uso che ne fa una multinazionale è diverso da quello che ne fa un politico, un'istituzione culturale o ancora un privato, soltanto per fare alcuni esempi ${ }^{40}$. Questa confusione, unita al fatto che il web solleva certamente alcuni problemi di qualità e di autenticità dei contenuti, non fa che alimentare una certa diffidenza di una parte del mondo accademico nei confronti dei nuovi media, sebbene negli anni più recenti si registri un'inversione di tendenza e si senta sempre più spesso parlare di «digital history» o più in generale di «digital humanities».

Nonostante il progetto CIRILI, come si è detto, non abbia creato un profilo social per la diffusione dei suoi contenuti, si è tuttavia stabilito di garantire l'accessibilità ai risultati del progetto con la preparazione di un libro bianco online, conformemente

37 Vedi per es. il progetto "Time Machine" sui Big Data del passato, candidato ad una Flagship nell'ambito dell'iniziativa "Future and Emerging Technologies" di H2020 (http://ec.europa.eu/programmes/horizon2020/en/h2020-section/future-and-emergingtechnologies).

${ }^{38} \mathrm{http}: / /$ pro.europeana.eu/about-us/our-vision

${ }^{39} \mathrm{http}: / /$ www.dariah.eu/about/dariah-in-a-nutshell.html

${ }^{40} \mathrm{G}$. Cosenza, Introdurione alla semiotica dei nuovi media, Roma-Bari 2014. 
alle esigenze dettate dall'Europa in tema di “open access". Una delle finalità del libro bianco inoltre è proprio quella di mostrare come un'indagine multidisciplinare che investe questioni complesse che investono la storia, l'archeologia, la tutela dell'ambiente e del patrimonio culturale, l'urbanizzazione, la formazione delle giovani generazioni debba promuovere l'uso di concetti imparziali, senza tuttavia dipingere un quadro roseo che occulti o attenui il carattere problematico. Per questo motivo una tappa importante e delicata è stata quella di raccogliere, organizzare e valutare le fonti relative al territorio, al patrimonio culturale e alle sue trasformazioni in modo logico e coerente secondo un metodo scientifico. Questa operazione preliminare è funzionale alla diffusione delle informazioni - costituite da documenti diversificati (testi, video, audio) che mirano all'oggettività - attraverso strumenti come il blog e i social media, in modo da coinvolgere un pubblico ampio (cittadini, politici, imprenditori, studenti) e non strettamente accademico. Soltanto accompagnando il lavoro sulla documentazione scientifica con una continua attività di mediazione culturale (attraverso seminari, dibattiti, visite, letture) è possibile coinvolgere e stimolare la società civile e in particolare le nuove generazioni alla comunicazione e discussione, affrontando tematiche complesse, come i concetti di cambiamento e di continuità, di comparazione e di confronto, di faziosità e di oggettività.

Già i primi risultati del progetto CIRILI permettono di intravedere che un suo naturale ulteriore sviluppo si inserisce bene nelle politiche dell'Unione Europea indicate nel Work Programme 2016-2017 di Horizon2020, dalla linea «Europe in a changing world - inclusive, innovative and reflective societies». Infatti i territori posti sul litorale e in aree deltizie sono spesso di difficile gestione per le amministrazioni, sia perché sono aree densamente popolate sia perché sono zone caratterizzate da lunghi processi di trasformazione storica del paesaggio naturale e del patrimonio culturale. Quando in queste aree si sviluppano attività economiche con un forte impatto ambientale, come un porto commerciale o un aeroporto (per es. il porto fluviale di Siviglia sul Guadalquivir o l'aeroporto Leonardo da Vinci a Fiumicino), è facile comprendere come si possano generare conflitti tra amministrazioni pubbliche, imprese private e cittadini che abitano nella zona. Sono i cittadini che vedono il territorio in cui vivono trasformarsi indipendentemente dalla loro volontà, a mobilitarsi e a costituire dei movimenti spontanei di protesta e contestazione. Del resto l'interesse prioritario dei politici, delle amministrazioni locali e delle imprese si realizza con difficoltà in investimenti sul territorio a lungo termine, che sono costosi e che non pagano in termini elettorali perché i risultati non si vedono nell'arco di un mandato politico o amministrativo. Di conseguenza le scelte politiche devono tener conto dell'esigenze dei cittadini che abitano in quel territorio e devono cercare di creare nuove opportunità di lavoro, senza provocare la lenta e continua distruzione del patrimonio ambientale e culturale. Tuttavia si tratta di un equilibrio difficile da gestire in aree così densamente urbanizzate dove l'agricoltura, il patrimonio naturalistico, l'archeologia finiscono per entrare inevitabilmente in conflitto con le esigenze di sviluppo delle infrastrutture: ne sono un esempio le modalità di sfruttamento del lago Mariout in Egitto, il progetto dell'autorità portuale di Siviglia di escavazione del Guadalquivir, il progetto di raddoppio dell'area dell'aeroporto di Fiumicino.

In questo contesto si colloca lo sviluppo del progetto CIRILI, che partendo dall'esperienza maturata in questo primo periodo di ricerca, ha offerto l'opportunità di studiare il territorio e promuovere attività diverse rispetto a quelle già esistenti, avvalendosi di una rete di partner locali e internazionali. Infatti i primi risultati del progetto hanno mostrato come CIRILI sia integrato nell'applicazione delle direttive 
del Consiglio Europeo, relative al patrimonio culturale come risorsa strategica per il futuro dell'Europa sostenibile: basti pensare all'organizzazione a maggio 2016 dell'atelier dottorale ad Alessandria (Egitto) che ha visto la partecipazione di giovani provenienti da tutto il bacino del Mediterraneo e che ha toccato temi fondamentali per lo sviluppo sostenibile offrendo un'innovativa chiave di lettura comparativa tra quattro territori deltizi, come si evince dal programma ${ }^{41}$.

Terminata con successo questa prima fase, il progetto CIRILI potrebbe proseguire la ricerca proponendosi come un modello per lo studio dei processi di evoluzione storica di lungo periodo includendo nella comparazione anche altri territori deltizi, come quello del Po, del Danubio e del Reno. L'obiettivo infatti è quello di estendere la prospettiva di ricerca sempre più a una dimensione internazionale e aperta ad accogliere le sollecitazioni esterne al singolo territorio oggetto di studio. Il lavoro dell'équipe CIRILI, interdisciplinare e internazionale, ha chiaramente mostrato che per ottenere risultati dallo studio di questi complessi territori è necessario uscire dai percorsi tradizionali. Come emerge dall'analisi comparativa dei quattro territori deltizi condotta nell'ambito del progetto CIRILI, lo studio del territorio in una prospettiva storica coinvolge tematiche molto ampie e complesse e non si può dunque limitare alla ricostruzione di un singolo edificio o ad una visita virtuale o reale che sia. Al centro della storia delle trasformazioni del territorio e del patrimonio naturale e culturale vi sono le persone che, direttamente o indirettamente, hanno contribuito a cambiarli e a renderli quello che sono adesso.

E' necessario quindi trasmettere questa consapevolezza alla società civile e al largo pubblico ed è proprio in questa direzione che le politiche dell'Europa spingono in tema di finanziamenti alla ricerca, quando insistono sulla "cittadinanza attiva", la "società inclusiva" e lo "sviluppo sostenibile". La sfida dell'équipe CIRILI è dunque quella di essere i primi promotori di un centro di studio dei territori deltizi sulla lunga durata, con una particolare attenzione alle problematiche attuali.

Ciò comporta di doversi avvalere di una metodologia comunicativa innovativa che coniughi la ricerca sulle trasformazioni del territorio con gli strumenti offerti dal web, in linea con le ultime tendenze della public bistory, corrente storiografica che ha l'intento di sviluppare un metodo e un dibattito su come divulgare la storia ad un pubblico ampio e non specialistico. Infatti la figura dello storico nel suo ruolo tradizionale di ricercatore, insegnante o mediatore deve necessariamente comprendere i cambiamenti disciplinari dovuti alle nuove tecnologie e riuscire a dominarli, se vuole continuare a svolgere con successo queste importanti funzioni all'interno della società. Si tratta quindi di una sfida importante che lo storico non può affrontare soltanto da un punto di vista teorico o come fruitore passivo dei contenuti digitali, ma è una sfida che deve vincere occupando uno spazio nel mondo dei nuovi media e del web. Il ricercatore, il curatore di un museo o l'insegnante, dovranno imparare a promuovere i propri progetti e a divulgarne i contenuti in modo tale che l'utilità sociale della ricerca storica sia maggiormente visibile, anche grazie all'uso dei nuovi media ${ }^{42}$.

${ }^{41}$ https:// cirili.hypotheses.org/532

${ }^{42} \mathrm{~S}$. Noiret, La digital history: histoire et mémoire à la portée de tous, in «Read/Write Book 2: une introduction aux humanités numériques », [en ligne], Marseille, OpenEdition Press, 2012, pp. $1-46$; cfr. anche D. Fiormonte, T. Numerico, F. Tomasi, The Digital Humanist. A critical Inquiry, New York 2015, p. 123-128. 
Non è un caso che negli ultimi anni la public bistory ha assunto un rilievo crescente nella società e ha ora un suo spazio e un riconoscimento all'interno delle società degli storici: ne sono una testimonianza l' "International Federation of Public History" (IFPH) nata nel 2013 e la neonata "Associazione Italiana di Public History" (AIPH), al cui primo congresso - che si è tenuto nel giugno 2017 a Ravenna - hanno partecipato moltissimi ricercatori, cittadini, imprese, studenti, operatori museali che in un modo o nell'altro si occupano di storia con un'attenzione particolare all'attualità e alle trasformazioni del territorio.

Proprio l'apertura della public history al dialogo con altre discipline e ai cambiamenti che i processi storici hanno sulla società e sul territorio attuale hanno indotto l'équipe CIRILI a presentare il progetto nella sezione Poster del IV Congresso della IFPH a giugno 2017 con il titolo: Public History and Cultural Heritage Management in the "City, River, Littoral" Project. Il progetto CIRILI è stato accolto con grande interesse mostrando che il lavoro finora svolto abbia notevoli prospettive di sviluppo in ambito nazionale e internazionale. Soltanto infatti percorrendo la via dell'innovazione si possono aprire nuove modalità per promuovere l'inclusione, la coesione sociale e il dialogo interculturale, nella direzione richiesta dalle politiche dell'Europa e i contributi raccolti in questo libro bianco ne sono una dimostrazione. 